\title{
Calorimetría indirecta en cuidado crítico: una revisión narrativa
}

\author{
Indirect calorimetry in critical care: A narrative review \\ Calorimetria indireta no cuidado crítico: uma revisão narrativa
}

\author{
Abel Salvador Arroyo-Sánchez ${ }^{1 *}$
}

Recibido: 22 de enero 2020. Aceptado para publicación: 22 de marzo 2020

Publicado en línea: 11 de julio de 2020

https://doi.org/10.35454/rncm.v3n2.88

\begin{abstract}
Resumen
Introducción: la subnutrición y la sobrenutrición del paciente en estado crítico se han asociado a resultados adversos, por ello es necesario contar con un instrumento que nos permita medir el gasto energético de forma individualizada y tener en cuenta los factores que pueden afectar esta medición.
\end{abstract}

Objetivo: revisar los conceptos básicos y aplicados de la calorimetría indirecta (CI), de la evidencia actual que sustenta su uso y de las perspectivas futuras en los cuidados críticos.

Método: se realizó una revisión narrativa, con búsqueda no sistemática de artículos relevantes en español e inglés utilizando las palabras clave calorimetría indirecta, cuidados críticos, soporte nutricional y metabolismo energético. Las bases de datos consultadas fueron Medline, PubMed, Scielo, BVS, Redalyc, Latindex y Google académico; incluyendo los manuscritos publicados en los últimos 15 años.

Resultados: la utilidad de la $\mathrm{Cl}$ ventilatoria como guía de las metas calóricas en pacientes en estado crítico es controversial por los resultados de los estudios TICACOS, SPN y EAT-ICU. A pesar de ello sigue siendo recomendado como el método de primera elección para calcular el gasto energético en estos pacientes, por ser el método más objetivo, confiable, práctico y personalizado.

\section{Summary}

Introduction: Undernutrition and overnutrition of the critically ill patient have been associated with adverse outcomes, so it is necessary to have an instrument that allows us to measure energy expenditure individually and taking into account the factors that can affect this measurement.

Objective: To review the basic and applied concepts of indirect calorimetry (IC), of the current evidence that supports its use and of future perspectives in critical care.

Method: A narrative review was performed, with a systematic search for relevant articles in Spanish and English using the keywords indirect calorimetry, critical care, nutritional support and energy metabolism. The databases consulted were Medline, PubMed, Scielo, BVS, Redalyc, Latindex and Google scholar; including manuscripts published in the last 15 years.

Result: The usefulness of ventilatory IC as a guide to caloric goals in critically ill patients is controversial because of the results of the TICACOS, SPN and EAT-ICU studies. Despite this, it is still recommended as the first choice method to calculate the energy expenditure in these patients, as it is the most objective, reliable, practical and personalized method.

Conclusion: The IC helps to manage goals, nutritional readjustment according

\section{Resumo}

Introdução: a subnutrição e a supernutrição de pacientes críticos têm sido associadas a resultados adversos, sendo necessário um instrumento que permita medir o gasto energético individualmente e levar em consideração os fatores que podem afetar essa medida.

Objetivo: rever os conceitos básicos e aplicados de calorimetria indireta $(\mathrm{Cl})$, as evidências atuais que sustentam seu uso e as perspectivas futuras em cuidados intensivos.

Método: foi realizada uma revisão narrativa, com busca sistemática de artigos relevantes em espanhol e inglês, utilizando as palavras-chave calorimetria indireta, cuidados intensivos, suporte nutricional e metabolismo energético. As bases de dados consultadas foram: Medline, PubMed, Scielo, BVS, Redalyc, Latindex e Google acadêmico; incluindo manuscritos publicados nos últimos 15 anos.

Resultados: a utilidade da Cl ventilatória como guia para objetivos calóricos em pacientes críticos é controversa devido aos resultados dos estudos TICACOS, SPN e EAT-ICU. Apesar disso, ainda é recomendado como o método de primeira escolha para o cálculo do gasto energético nesses pacientes, pois é o método mais objetivo, confiável, prático e personalizado. 
Conclusión: la $\mathrm{Cl}$ ayuda a realizar un manejo dirigido a cumplir objetivos, a hacer el reajuste nutricional de acuerdo con la evolución clínica de forma individualizada, y así evitar la sub nutrición o la sobre nutrición.

Palabras clave: calorimetría indirecta, cuidados críticos, soporte nutricional, metabolismo energético.

Universidad Privada Antenor Orrego, Hospital Víctor Lazarte Echegaray, Trujillo, Perú. to the clinical evolution individually and thus avoid undernutrition or overnutrition.

Keywords: Indirect Calorimetry; Critical care; Nutritional support; Energy metabolism.
Conclusão: o $\mathrm{Cl}$ ajuda a realizar um manejo voltado ao cumprimento dos objetivos, realizar reajustes nutricionais de acordo com a evolução clínica individual e, assim, evitar desnutrição ou supernutrição.

Palavras-chave: calorimetria indireta, cuidados intensivos, suporte nutricional, metabolismo energético.

\section{INTRODUCCIÓN}

El soporte nutricional es indispensable para la recuperación de cualquier paciente en estado crítico $^{(1)}$. El aporte energético es obtenido de los macronutrientes (carbohidratos, proteínas y lípidos) y la proporción de cada uno de ellos debe ser balanceada así como estar de acuerdo con las necesidades específicas del organismo durante la evolución de la enfermedad que lo aqueja ${ }^{(1,2)}$. Actualmente se recomienda la práctica de una medicina dirigida a objetivos puntuales y a su vez personalizada o individualizada ${ }^{(1-3)}$. Además, cada vez es más factible disponer de instrumentos de apoyo clínico que se pueden utilizar en la cabecera del paciente y que nos permite la toma de decisiones inmediatas para el ajuste de nuestro tratamiento (POCT: Point of care testing) ${ }^{(4)}$.

La administración de soporte energético óptimo y personalizado se considera necesaria ya que se ha descrito que un aporte cercano a $70 \%$ del requerimiento energético en los pacientes en estado crítico está asociado a menor mortalidad, mientras que un aporte superior a $100 \%$ estuvo asociado a mayor mortalidad. De esto se concluye que la baja nutrición y la sobre nutrición pueden ser deletéreas para el paciente en estado crítico $^{(5)}$. Este resultado adverso parece ser más evidente en los pacientes con desnutrición previa al ingreso a la unidad de cuidados intensivos $(\mathrm{UCI})^{(6)}$.

Los requerimientos energéticos entre las personas sanas son diferentes debido a múltiples factores: morfológicos, endocrinológicos, metabólicos, medicamentos usados, de actividad física y medioambientales (Figura 1 y Tabla 1); estas diferencias serán más importantes en los pacientes que se encuentren en estado crítico $^{(7)}$. Una herramienta en nutrición clínica que cumple con

las características de cuantificar el requerimiento energético individualizado en un momento específico es la calorimetría indirecta (CI).

El objetivo de la presente revisión es actualizar los conceptos básicos y aplicados de la calorimetría indirecta, que sustenta su uso y de las perspectivas futuras en los cuidados críticos.

\section{MÉTODO}

Se realizó una revisión narrativa, con búsqueda no sistemática de artículos relevantes en español e inglés utilizando las palabras clave calorimetría indirecta, cuidados críticos, soporte nutricional y metabolismo energético. Las bases de datos consultadas fueron Medline, PubMed, Scielo, BVS, Redalyc, Latindex y Google académico; incluyendo los manuscritos publicados en los últimos 15 años.

\section{ANÁLISIS Y DISCUSIÓN}

El gasto energético total (GET) diario en una persona sana corresponde a la suma de tres componentes: el gasto energético basal (energía necesaria para que el corazón lata, el diafragma se contraiga, se sinteticen y excreten hormonas y jugos intestinales, etc.), la termogénesis inducida por la dieta (energía necesaria para ingerir, degradar y absorber los alimentos) y la actividad física desarrollada. La suma del gasto energético basal (GEB) más la termogénesis, se denomina gasto energético en reposo (GER) ${ }^{(7-12)}$. En el paciente en estado crítico se considera que la actividad física desarrollada por el individuo será reemplazada por la severidad de la enfermedad que lo aqueja (por ejemplo, postoperatorio agudo o 


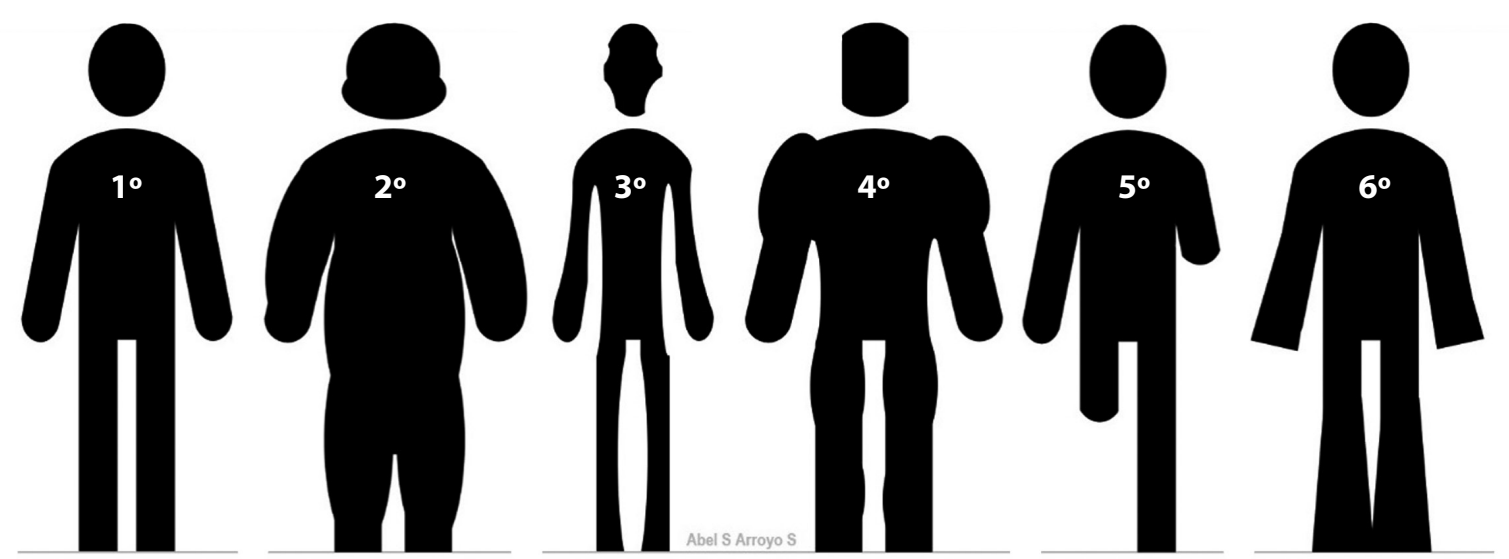

Figura 1. Variabilidad morfológica y gasto energético. Todos los pacientes tienen la misma talla y edad siendo el $1^{\circ}$ de ellos eutrófico para su talla, el $2^{\circ}$ obeso, el $3^{\circ}$ emaciado, el $4^{\circ}$ hipertrófico, el $5^{\circ}$ amputado y el $6^{\circ}$ edematoso. Por lo tanto, la composición corporal y el requerimiento energético de cada paciente serán diferentes a pesar de tener la misma edad y estatura.

Tabla 1. Factores fisiológicos, patológicos y farmacológicos que intervienen en el gasto energético total (GET) del paciente en estado crítico ${ }^{(8,14)}$

\begin{tabular}{|l|l|}
\hline \multicolumn{1}{|c|}{ Aumentan el GET } & \multicolumn{1}{c|}{ Disminuyen el GET } \\
\hline Sexo masculino & Sexo femenino \\
\hline Crecimiento & Envejecimiento \\
\hline Tipo de enfermedad & Tipo de enfermedad \\
\hline Fiebre $\left(+7 \%\right.$ por cada $\left.1^{\circ} \mathrm{C}\right)$ & Hipotermia $\left(-7 \%\right.$ por cada $\left.1^{\circ} \mathrm{C}\right)$ \\
\hline Dolor & Drogas: sedantes, opiáceos, $\beta$-bloqueadores, miorrelajantes \\
\hline Movimientos patológicos & Desnutrición, ayuno \\
\hline Drogas: vasopresores, antineoplásicos & Hipoventilación, tipo de ventilación mecánica \\
\hline Cuidados y actividades de enfermería & \\
\hline Sobrealimentación o nutrientes en bolo & \\
\hline Hiperventilación, tipo de ventilación mecánica, desconexión & \\
\hline
\end{tabular}

electivo, sepsis, trauma, quemadura, etc. $)^{(8,9,11)}$, como se muestra en la Figura 2. Un factor que actualmente debe ser tomado en cuenta como generador de mayor GET es la terapia física a la que es sometido el paciente en estado crítico (incluso estando en ventilación mecánica) y en cuya medición deberemos tener en cuenta, la frecuencia de la terapia física a la que es sometido el paciente y si esta es pasiva o activa.

Los macronutrientes (carbohidratos, proteínas y lípidos) son la principal fuente de energía orgánica y cada uno de estos macronutrientes necesita ser oxidado lo que genera un consumo de oxígeno $\left(\mathrm{VO}_{2}\right)$ para pro- porcionar energía (Kcal) y producción de dióxido de carbono $\left(\mathrm{VCO}_{2}\right)$; la cantidad de oxígeno consumido por cada gramo de macronutriente, así como la cantidad de dióxido de carbono y kilocalorías que produce ese gramo, pueden verse detallados en la Tabla $2^{(9)}$.

\section{ORIGEN Y TIPOS DE CALORIMETRÍA}

Como lo describen Heymsfield et al.(7) en una revisión histórica sobre el recambio energético humano, la asociación empírica del calor y el mantenimiento de la vitalidad tuvo sus inicios en la época de Hipócrates y 


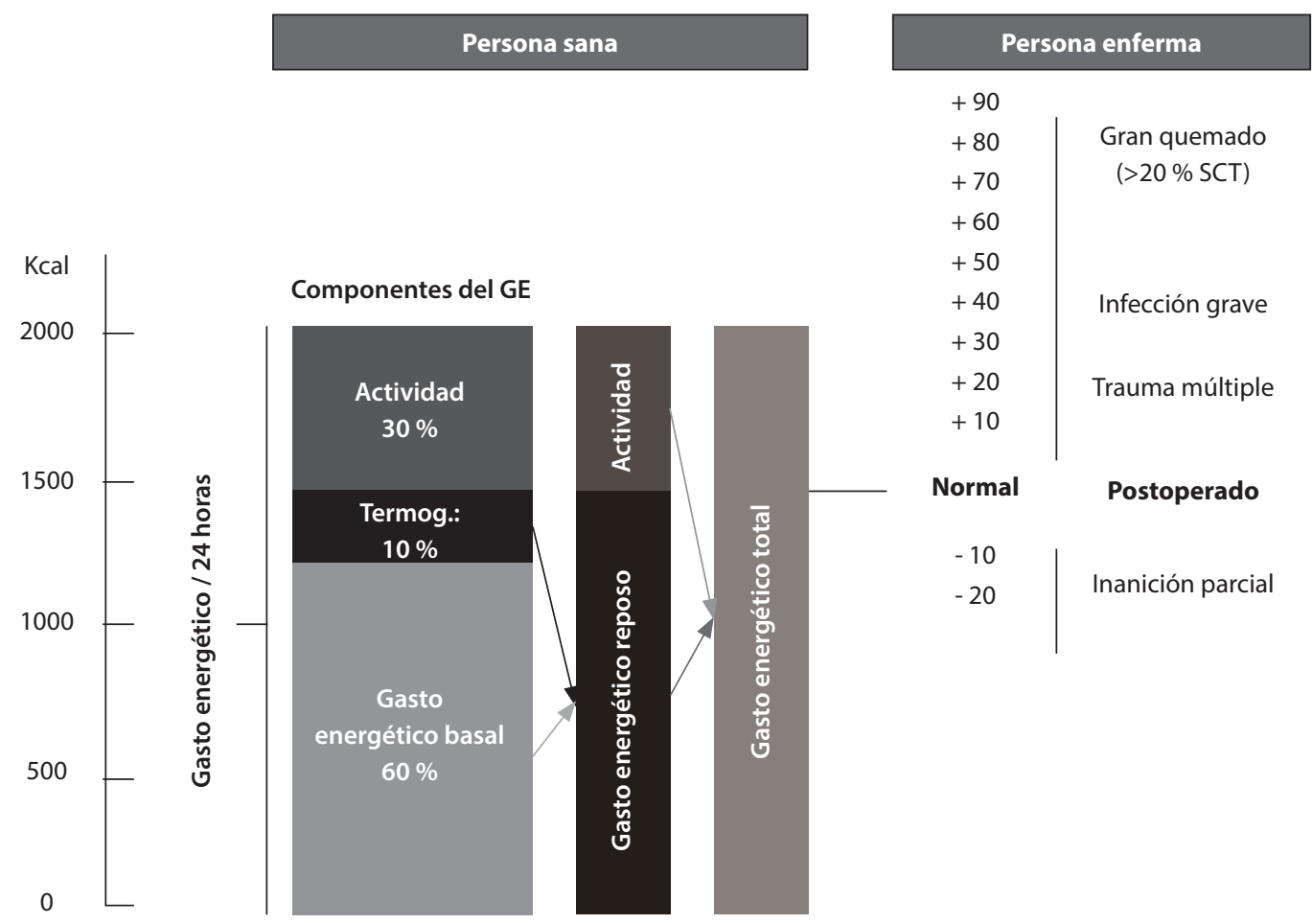

Figura 2. Componentes del gasto energético total en 24 horas (GET) de una persona sana (a la izquierda) y de una persona enferma (a la derecha). El GE en reposo se mantiene igual para ambos, pero en la persona enferma la actividad es reemplazada por la severidad de la enfermedad o disminuirá en casos de inanición (por ejemplo, la anorexia patológica). Termog:Termogénesis. SCT: Superficie corporal total. Modificado de: José Luis Pereira Cunill y Manuela Garrido Vázquez ${ }^{(26)}$. Interpretación de la Calorimetría Indirecta. Disponible en: https://es.slideshare.net/jlpc1962/interpretacion-de-la-calorimetra-indirecta.

Tabla 2. Metabolismo oxidativo de los macronutrientes (adaptado de Marino, et al ${ }^{(9)}$ )

\begin{tabular}{|cccccccc|}
\hline Macronutriente & & $\mathbf{V O}_{\mathbf{2}} \mathbf{( L / g )}$ & & $\mathbf{V C O}_{\mathbf{2}} \mathbf{( L / g )}$ & & Energía $(\mathbf{K c a l} / \mathbf{g})$ & $\mathbf{C R}$ \\
\hline Lípidos & + & 2,00 & $=$ & 1,40 & + & 9,0 & 0,70 \\
\hline Proteínas & + & 0,96 & $=$ & 0,78 & + & 4,0 & 0,82 \\
\hline Carbohidratos & + & 0,74 & $=$ & 0,74 & + & 3,7 & 1,00 \\
\hline
\end{tabular}

$\mathrm{VO}_{2}(\mathrm{~L} / \mathrm{g})$ : Consumo de oxígeno en litros por cada gramo. $\mathrm{VCO}_{2}(\mathrm{~L} / \mathrm{g})$ : Producción de dióxido de carbono en litros por cada gramo. Kcal / g: Kilocalorías generadas por la oxidación de cada gramo. CR: Cociente respiratorio.

su "teoría de los 4 humores". Posteriormente, Robert Boyle tras colocar un ratón y una vela en un frasco sellado y ver la forma como el ratón expiró y la vela se apagó casi al mismo tiempo, estableció una equivalencia de vida y fuego como procesos de combustión y concluyó que se necesitaba un elemento en el aire para mantener la respiración y la combustión. Ahora sabemos que ese componente es el oxígeno. Casi un siglo después, Lavoisier identificó que los seres vivos después de consumir oxígeno producían de manera proporcional calor y una nueva sustancia, que ahora sabemos que es el dióxido de carbono. Basándose en la $1^{\text {a }}$ Ley de la Termodinámica de von Mayer: "la energía no se puede crear ni destruir" y en que "la oxidación de los alimentos es la principal fuente de energía para los seres vivos", se inició la búsqueda de instrumentos que puedan medir el calor generado por un ser viviente en situaciones de reposo o actividad física (Figura 3 ).

Entre los instrumentos que nos permiten medir el GE del ser humano tenemos la calorimetría directa, considerada como el método más preciso para determinar el GER ${ }^{(8,11,13)}$. Consiste en colocar a la persona 


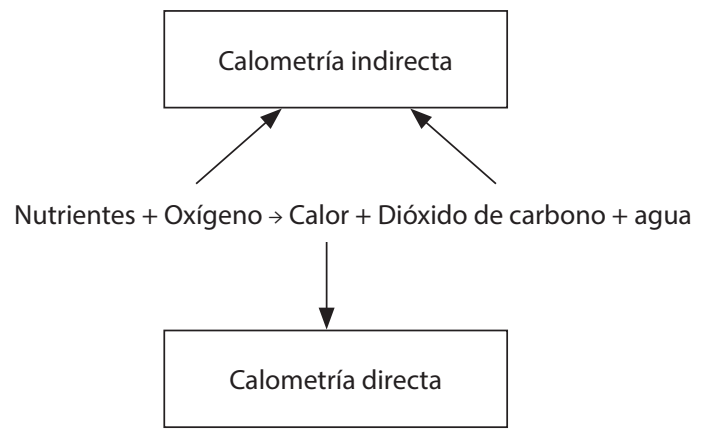

Figura 3. La calorimetría directa mide el gasto energético a través del calor generado, mientras que la calorimetría indirecta lo hace a través de la medición de la concentración de oxígeno que se consume $\left(\mathrm{VO}_{2}\right)$ y del dióxido de carbono que se produce $\left(\mathrm{VCO}_{2}\right)^{(11)}$.

dentro de una cámara aislada y sellada, donde se cuantifica el calor generado por el individuo a través de los cambios de temperatura del aire y el agua que ingresan y salen de la cámara (Figura 3). Lamentablemente, es un método costoso, que requiere un equipo complejo y no es práctico, en el caso de pacientes en estado crítico, por lo que en la actualidad su principal uso es en investigación $^{(8,11,13)}$.

Una alternativa a la calorimetría directa es la CI, la cual usa la medición de la concentración de oxígeno y de dióxido de carbono para, a través de ecuaciones matemáticas, calcular el gasto energético. La CI puede ser circulatoria o ventilatoria ${ }^{(8,13)}$.

La CI circulatoria, calcula el gasto energético usando la saturación de oxígeno arterial y la saturación de dióxido de carbono de la sangre venosa mixta (obtenida por un catéter en la arteria pulmonar), y aplicando la ecuación de Fick ${ }^{(8,13)}$ :

$$
\mathrm{GE}=\mathrm{GC} \times \mathrm{Hb}\left(\mathrm{S}_{\mathrm{a}} \mathrm{O}_{2}-\mathrm{S}_{\mathrm{v}} \mathrm{O}_{2}\right) \times 95,18
$$

GE: Gasto energético. GC: Gasto cardiaco (obtenido por termodilución). $\mathrm{Hb}$ : Hemoglobina. $\mathrm{S}_{\mathrm{a}} \mathrm{O}_{2}$ : Saturación arterial de oxígeno. $\mathrm{S}_{\mathrm{v}} \mathrm{O}_{2}$ : Saturación venosa mixta de oxígeno.

A pesar de ser un método prometedor, en el paciente en estado crítico su exactitud disminuye, debido a que son pacientes hemodinámicamente inestables y con patología pulmonar frecuente, además de ser un método invasivo, realiza dicha medición en un solo momento (el de la toma de muestra de gases sanguíneos y del gasto cardiaco) ${ }^{(8,13)}$.
La CI ventilatoria, que es motivo de la presente revisión y en adelante nos referiremos exclusivamente a ella, es un método que se basa en el cálculo del GER a través de la fórmula de Weir, donde se mide el $\mathrm{VO}_{2}$, la $\mathrm{VCO}_{2}$ y la pérdida urinaria de nitrógeno $\left(\mathrm{N}_{\mathrm{u}}\right)^{(6,8-14)}$ :

$$
\begin{gathered}
\mathrm{GE}(\mathrm{Kcal} / \text { día })=\left[3,94\left(\mathrm{VO}_{2} \mathrm{~mL} / \mathrm{min}\right)+\right. \\
\left.1,11\left(\mathrm{VCO}_{2} \mathrm{~mL} / \mathrm{min}\right)\right] 1,44-2,17\left(\mathrm{~N}_{\mathrm{u}} \mathrm{g} / \mathrm{d}\right)
\end{gathered}
$$

Como la proporción de GE obtenido por la $\mathrm{N}_{\mathrm{u}}$ es menor a $4 \%-5 \%$ del GE total, se puede omitir la inclusión de este parámetro en el cálculo final (ecuación de Weir simplificada $)^{(9-11)}$ :

$$
\begin{gathered}
\mathrm{GE}(\mathrm{Kcal} / \text { día })=\left[3,94\left(\mathrm{VO}_{2} \mathrm{~mL} / \mathrm{min}\right)+\right. \\
\left.1,11\left(\mathrm{VCO}_{2} \mathrm{~mL} / \mathrm{min}\right)\right] 1,44
\end{gathered}
$$

Adicionalmente, la CI permite calcular el Cociente Respiratorio (CR) que es la relación existente entre la $\mathrm{VCO}_{2} / \mathrm{VO}_{2}$; donde un metabolismo equilibrado estará entre los rangos de 0,7 a 1,10; mientras que un valor $<0,7$ significa lipólisis y un valor $>1,10$ indica lipogénesis por exceso de carbohidratos, como se puede apreciar en la Tabla $2^{(8-15)}$.

Existen factores fisiopatológicos del paciente y algunos medicamentos administrados que pueden aumentar o disminuir el GE al momento de realizar la CI, los mismos que se encuentran resumidos en la Tabla 1 y deben ser tomados en cuenta a la hora de hacer la medición $^{(8,15)}$; así mismo se deben considerar los factores técnicos que especifique el fabricante del equipo de $\mathrm{CI}^{(8,10,11)}$. Es por lo antes mencionado que se propone una lista de chequeo con los requisitos que deben cumplirse, en su totalidad, antes de hacer la medición como se puede apreciar en la Tabla 3.

Entre los pacientes de la UCI que se beneficiarían de la realización de la CI consideramos las indicaciones propuestas por Mtaweh et al. ${ }^{(11)}$, la American Association for Respiratory Care $^{(14)}$ y Delsoglio et al. ${ }^{(15)}$, las cuales son: pacientes con morfología alterada (amputados, obesos, edematosos), pacientes con estrés metabólico severo (sepsis, pancreatitis grave, trauma multisistémico, gran quemado), gestantes, pacientes con signos clínicos de pobre o sobre nutrición (retiro difícil) y pacientes con inadecuada respuesta a las ecuaciones predictivas (sin mejoría de parámetros bioquímicos). Por otro lado, la CI debe ser evitada en pacientes con las siguientes contraindicaciones: con fuga endotraqueal o en el circuito ventilatorio, pacientes con fístula 
Tabla 3. Lista de chequeo de los requisitos que deben cumplirse para realizar la calorimetría indirecta utilizada en el Hospital Víctor Lazarte Echegaray del Perú

\begin{tabular}{|c|c|c|c|}
\hline $\mathbf{N}^{\circ}$ & Criterio para evaluar & Si & No \\
\hline 1 & El paciente ha estado en reposo en decúbito supino, mínimo durante 30 minutos antes del estudio. & & \\
\hline 2 & $\begin{array}{l}\text { Se ha suspendido la nutrición enteral (NE) o nutrición parenteral (NP) continua o intermiente dos horas antes del } \\
\text { estudio. }\end{array}$ & & \\
\hline 3 & El ritmo y la composición de la NE o NP continua se han mantenido durante las12 horas previas al estudio. & & \\
\hline 4 & La temperatura ambiente se mantiene en cerca de $25^{\circ} \mathrm{C}$. & & \\
\hline 5 & Los parámetros ventilatorios se mantienen hasta mínimo 90 minutos antes del estudio. & & \\
\hline 6 & La fracción inspiratoria de oxígeno $\left(\mathrm{FiO}_{2}\right)$ debe mantenerse constante durante todo el estudio. & & \\
\hline 7 & La sedoanalgesia es adecuada por lo menos 30 minutos antes del estudio. & & \\
\hline 8 & Las dosis de vasopresores e inotrópicos se mantienen sin modificación por lo menos 2 horas antes del estudio. & & \\
\hline 9 & $\begin{array}{l}\text { No recibir anestesia general dentro de las } 6-8 \text { horas, TRR dentro de las 3-4 horas o procedimiento doloroso en la } \\
\text { última hora. }\end{array}$ & & \\
\hline 10 & Los cuidados de enfermería se suspendieron 30 minutos antes, y se suspenderán durante el desarrollo del estudio. & & \\
\hline 11 & $\begin{array}{l}\text { Hay mediciones en "equilibrio", es decir: en } 5 \text { minutos, las variaciones de } \mathrm{VO}_{2} \text { y } \mathrm{VCO}_{2} \text { son }<10 \% \text { y los cambios del } \\
\text { CR son }<5 \% \text {. }\end{array}$ & & \\
\hline
\end{tabular}

TRR: Terapia de reemplazo renal. $\mathrm{VO}_{2}$ : Consumo de oxígeno. $\mathrm{VCO}_{2}$ : Producción de dióxido de carbono.

broncopleural, con presión positiva al final de la espiración $(\mathrm{PEEP})>12 \mathrm{mmHg}$ y pacientes que requieren una fracción inspiratoria de oxígeno $\left(\mathrm{FiO}_{2}\right)>0,6^{(8,11,13-15)}$.

A continuación, se enumeran los requisitos previos a la realización de la CIy la duración de la medición ${ }^{(8,10-15)}$ :

1. Condiciones y preparación del calorímetro

- Fase de calentamiento o puesta a punto, calibración del neumotacógrafo y los analizadores de gas a $21 \%$ (oxígeno ambiental)

2. Preparación del paciente y condiciones

- Paciente en reposo decúbito supino por 30 minutos antes del estudio

- Que hayan pasado 2 horas de la última ingesta o del cese de la nutrición artificial

- En pacientes con nutrición continua no modificar ritmo o composición de la nutrición durante las 12 horas previas

- Ambiente con temperatura aproximada de $25^{\circ} \mathrm{C}$

- No modificar los parámetros ventilatorios por lo menos 90 minutos antes

- La fracción inspiratoria de oxígeno debe mantenerse constante durante todo el estudio

- Optimizar la sedoanalgesia por lo menos 30 minutos antes del estudio
- Si necesita usar drogas vasopresoras o inotrópicos, mantener la misma dosis hasta mínimo 2 horas antes del estudio

- No haber recibido anestesia general por lo menos 6 - 8 horas, ni hemodiálisis al menos 3 - 4 horas, ni procedimiento doloroso al menos una hora antes

- Los cuidados de enfermería y de rutina deben suspenderse durante el estudio

- Las mediciones durante el estado de equilibrio son representativas de las 24 horas; se considera "equilibrio" cuando en 5 minutos las variaciones del $\mathrm{VO}_{2}$ $\mathrm{y} \mathrm{VCO}_{2}$ son $<10 \%$ y los cambios de CR son $<5 \%$.

3. Duración de la medición

- La calorimetría debe realizarse en aproximadamente 30 minutos y se puede realizar 1 o 2 veces al día, siempre y cuando la ejecución del estudio no interfiera con el tratamiento intensivo requerido por el paciente en estado crítico.

Oshima et al. ${ }^{(8)}$ recomiendan realizar la primera medición a partir del $3^{\text {er }}-4^{\text {to }}$ día de ser admitido en la UCI (idealmente, cuando esté compensado), luego se puede repetir cada 2 a 3 días durante su estancia en la UCI o repetir antes en caso de que surjan cambios en 
las condiciones del paciente o de la enfermedad que lo aqueja.

El registro de la medición debe hacerse en un formato que permita ver de forma panorámica los factores que pueden estar afectando los resultados obtenidos al momento de comparar su evolución. La Figura 4, muestra el formato usado para este fin en el Hospital Víctor Lazarte Echegaray del Perú.

\section{INTERPRETACIÓN}

La interpretación debe hacerse teniendo en cuenta el estado fisiopatológico del paciente al momento de realizar la medición y los medicamentos recibidos. Mtaweh et al. ${ }^{(11)}$ proponen una tabla en la que describe los resultados obtenidos en el $\mathrm{VO}_{2}$, la $\mathrm{VCO}_{2}$ y el CR, así como las combinaciones de estos resultados y las posibles causas de las diferentes variaciones de las mediciones encontradas (Tabla 4).

\section{EVIDENCIA ACTUAL}

La Sociedad Americana de Nutrición Parenteral y Enteral (American Society for Parenteral and Enteral
Nutrition, ASPEN) en su guía para medir y proveer soporte nutricional en el adulto en estado crítico publicada en el año $2016^{(2)}$; sugiere calcular el GE en el paciente en condición crítica por medio de la CI, cuando el instrumento esté disponible y en ausencia de factores que afecten su exactitud. Así mismo, y basados en una opinión de expertos, ante la falta de CI, sugieren el uso de ecuaciones predictivas (EP) basadas en el peso $(25-30 \mathrm{Kcal} / \mathrm{Kg} /$ día $)$ para determinar el GE. Sin embargo, la validación de las diferentes EP usando la CI como estándar de oro no ha dado buenos resultados. Zusman et al. ${ }^{(16)}$ intentaron validar las EP más comúnmente usadas (25 Kcal / kg / día, Harris-Benedict con y sin factor de corrección, Penn State University, IretonJones, Faisy, Mifflin-St. Jeor y Jolliet) en 1440 pacientes encontrando que el nivel de precisión de las ecuaciones no superó $50 \%$ en los pacientes de cuidados intensivos.

A fines del año 2018, Heyland \& Stoppe ${ }^{(17)}$ realizaron una actualización de su revisión sistémica sobre el uso de la CI versus las EP en nutrición enteral (NE), sin encontrar estudios recientes que modifiquen su postura al respecto; concluyen que en pacientes quemados ${ }^{(18)} \mathrm{el}$ uso de la CI comparada con la EP de Curreri en la guía

\begin{tabular}{|c|c|c|c|c|c|c|c|c|c|c|}
\hline $\mathrm{N}^{0}$ & \multicolumn{5}{|c|}{ Apellidos y Nombre } & \multicolumn{2}{|l|}{$\mathrm{N}^{*} \mathrm{HC}:$} & \multirow[b]{4}{*}{1} & \multirow[b]{4}{*}{1} & \\
\hline \multicolumn{2}{|l|}{ Edad: } & Talla: & & Peso: & & & & & & \\
\hline \multicolumn{2}{|c|}{ F.Ingreso $\mathrm{H}$ : } & F.Ingreso U & & APACHE II & & \multicolumn{2}{|l|}{ SOFAi: } & & & \\
\hline Fecha & & 1 & 1 & 1 & 1 & 1 & 1 & & & \\
\hline TAM & $\mathrm{mmHg}$ & & & & & & & & & \\
\hline$T^{0}$ & ${ }^{\circ} \mathrm{C}$ & & & & & & & & & \\
\hline FR & $\mathrm{rpm}$ & & & & & & & & & \\
\hline $\mathrm{FC}$ & Ipm & & & & & & & & & \\
\hline Dopam & $\mathrm{ug} / \mathrm{K} / \mathrm{min}$ & & & & & & & & & \\
\hline Noradr & $\mathrm{ug} / \mathrm{K} / \mathrm{min}$ & & & & & & & & & \\
\hline Dobut & $\mathrm{ug} / \mathrm{K} / \mathrm{min}$ & & & & & & & & & \\
\hline Midaz & ug/K/min & & & & & & & & & \\
\hline Fenta & $\mathrm{ug} / \mathrm{K} / \mathrm{min}$ & & & & & & & & & \\
\hline Vecur & $\mathrm{ug} / \mathrm{K} / \mathrm{min}$ & & & & & & & & & \\
\hline Nebol & $\mathrm{kc} / \mathrm{d}$ & & & & & & & & & \\
\hline Necon & $\mathrm{kc} / \mathrm{d}$ & & & & & & & & & \\
\hline NPT & $\mathrm{kc} / \mathrm{d}$ & & & & & & & & & \\
\hline NutMx & $\mathrm{kc} / \mathrm{d}$ & & & & & & & & & \\
\hline NPO & $\mathrm{kc} / \mathrm{d}$ & & & & & & & & & \\
\hline \multicolumn{10}{|l|}{ ModVM } & \\
\hline Vol Min & $\mathrm{ml} / \mathrm{min}$ & & & & & & & & & \\
\hline PEEP & $\mathrm{cmH} 2 \mathrm{O}$ & & & & & & & & & \\
\hline $\mathrm{VO} 2$ & $\mathrm{mil} / \mathrm{min}$ & & & & & & & & & \\
\hline $\mathrm{vCO} 2$ & $\mathrm{mil} / \mathrm{min}$ & & & & & & & & & \\
\hline GER & $\mathrm{Ke} / \mathrm{d}$ & & & & & & & & & \\
\hline CR & & & & & & & & & & \\
\hline
\end{tabular}

Figura 4. Formato de registro de las mediciones por calorimetría indirecta utilizada en el Hospital Víctor Lazarte Echegaray de Perú. Incluye los parámetros clínicos, nutricionales, farmacológicos y ventilatorios al momento de realizar la medición, los que ayudarán a interpretar las variaciones en los resultados obtenidos en el contexto clínico del momento en que se realizó el estudio. 
Tabla 4. Interpretación de los resultados de la calorimetría indirecta ${ }^{(11)}$

\begin{tabular}{|l|l|l|}
\hline \multicolumn{1}{|c|}{ Resultados } & \multicolumn{2}{|c|}{ Causa probable } \\
\hline Elevación del $\mathrm{VCO}_{2}$ y del CR & $\begin{array}{l}\text { Acidosis metabólica } \\
\text { Hiperventilación }\end{array}$ & $\begin{array}{l}\text { Hipermetabolismo } \\
\text { Excesiva ingesta carbohidratos }\end{array}$ \\
\hline Disminución del $\mathrm{VCO}_{2}$ y del CR & $\begin{array}{l}\text { Alcalosis metabólica } \\
\text { Hipometabolismo } \\
\text { Inanición / Cetosis } \\
\text { Oxidación de etanol }\end{array}$ & $\begin{array}{l}\text { Hipoventilación } \\
\text { Hipoalimentación } \\
\text { Gluconeogénesis } \\
\text { Fuga de aire }\end{array}$ \\
\hline Elevación del $\mathrm{VO}_{2}$ & $\begin{array}{l}\text { Sepsis } \\
\text { Hipermetabolismo } \\
\text { Aumento ventilación/min } \\
\text { Escalofríos / agitación / tremores }\end{array}$ & $\begin{array}{l}\text { Hemodiálisis (<4 horas del procedimiento) } \\
\text { Sobrealimentación } \\
\text { Transfusión sanguínea } \\
\text { Hipertermia }\end{array}$ \\
\hline Disminución del VO & $\begin{array}{l}\text { Hipotermia } \\
\text { Parálisis } \\
\text { Ayuno / inanición } \\
\text { Anestesia general }\end{array}$ & $\begin{array}{l}\text { Hipotiroidismo } \\
\text { Sedación profunda } \\
\text { Edad avanzada } \\
\text { Coma / sueño profundo }\end{array}$ \\
\hline
\end{tabular}

$\mathrm{VO}_{2}$ : Consumo de oxígeno. $\mathrm{VCO}_{2}$ : Producción de dióxido de carbono. $\mathrm{CR}$ : Cociente respiratorio.

de la NE, no ha tenido efecto sobre la mortalidad; pero cuando la CI es usada como guía de la NE suplementada con nutrición parenteral (NP), en pacientes en estado crítico ventilados ${ }^{(19)}$, ha conseguido disminuir la mortalidad hospitalaria, aunque estos resultados se asociaron a mayor tasa de infecciones, de estancia en la UCI y de duración de la ventilación mecánica.

La Sociedad Europea de Nutrición Clínica y Metabolismo (European Society for Clinical Nutrition and Metabolism, ESPEN) en su guía de Nutrición Clínica en la Unidad de Cuidados Intensivos ${ }^{(3)}$ recomienda, con un consenso fuerte, que para el paciente en estado crítico en ventilación mecánica se debe usar la CI para determinar el GE. Pero, si la CI no está disponible, por consenso recomiendan que se calcule el GE con el $\mathrm{VO}_{2}$ obtenido a través del catéter de arteria pulmonar o usar la $\mathrm{VCO}_{2}$ obtenida del ventilador mecánico que disponga de esta medición ${ }^{(20)}$, antes que usar las EP para tener una mejor evaluación del GE. Así mismo, en el caso del paciente obeso en estado crítico recomienda como una buena práctica y por consenso que el aporte energético debe ser guiado por la CI, y si la CI no está disponible, el aporte debe basarse en el peso corporal ajustado.

Las recomendaciones hechas por la $\operatorname{ASPEN}^{(2)}$ y ESPEN $^{(3)}$ sobre la CI, se basan en el resultado del estudio TICACOS (Tight calorie control study) $)^{(19)}$ y a resultados de metaanálisis que han encontrado escasa correlación de las EP con la CI.
El estudio TICACOS ${ }^{(19)}$ fue un ensayo randomizado, controlado, no ciego, en pacientes con ventilación mecánica de un hospital en Israel que buscó determinar si el soporte nutricional guiado por CI mejoraba los resultados clínicos cuando es comparado con una EP (25 Kcal / kg / día), para llegar a los objetivos nutricionales determinados se usó NE y NP complementaria de ser necesario. El aporte calórico, proteico y el balance energético diarios fueron mayores en los pacientes guiados por CI. La mortalidad hospitalaria, después de una análisis por intención de tratar, tuvo una tendencia a ser menor en el grupo de CI, sin llegar a ser significativa (32,3\% versus $47,7 \%$; $p=0,058)$; pero en cuanto a la mortalidad hospitalaria, por protocolo recibido, si se evidenció una reducción significativa $(28,5 \%$ versus $48,2 \% ; \mathrm{p}=0,023)$. Por otro lado, los pacientes del grupo de CI tuvieron mayor número de días en ventilación mecánica $(16,1 \pm 14,7$ días versus $10,5 \pm 8,3$ días; $p=0,03$ ), mayor estancia en la UCI $(17,2 \pm 14,6$ días versus $11,7 \pm 8,4$ días; $p=0,04)$ y una tendencia a mayores casos de neumonía asociada al ventilador $(27,7 \%$ versus $13,8 \%$; $=0,08)$.

Heidegger et al. ${ }^{(21)}$ realizaron en ensayo, randomizado y controlado en 305 pacientes ventilados en dos unidades de cuidados intensivos en Suiza, eran pacientes que al tercer día de estancia en la UCI no habían alcanzado $60 \%$ de su requerimiento energético calculado por EP. A partir del tercer día, los pacientes fueron randomizados en dos grupos, el grupo de estudio 
recibió NE más NP complementaria para alcanzar su requerimiento energético, el que ahora era calculado por CI, y el grupo de pacientes control que continuó con NE teniendo como objetivo energético el cálculo hecho por la EP. El periodo de intervención fue del $4^{\circ} \mathrm{al}$ $8^{\circ}$ día y luego se hizo el seguimiento desde el $9^{\circ}$ hasta el $28^{\circ}$ día. Los autores encontraron que el aporte calórico fue mayor en el grupo de NP complementaria guiado por CI versus NE guiado por EP durante el periodo de la intervención (28 Kcal / kg / día versus $20 \mathrm{Kcal} / \mathrm{kg}$ / día); entre el $9^{\circ}$ y $28^{\circ}$ días, el grupo de NP complementaria tuvo menos tasa de infección nosocomial que el de NE ( $27 \%$ versus $38 \%$; $=0,034)$, no se encontraron diferencias en otros resultados clínicos como los días de estancia, días en ventilación mecánica, tasa de mortalidad e hiperglucemia.

Allingstrup et al. ${ }^{(22)}$ realizaron un ensayo randomizado, estratificado, con grupo paralelo en 199 pacientes ventilados mecánicamente en un hospital danés para determinar si la nutrición temprana dirigida a objetivos medidos por CI y la excreción de urea en orina de 24 horas mejoraban la calidad de vida física a los 6 meses de seguimiento, comparada con la nutrición estándar (25 Kcal / kg / día). Es importante mencionar que en este estudio se excluyeron pacientes con índice de masa corporal $<17 \mathrm{Kg} / \mathrm{m}^{2}$ y los que al parecer estaban desnutridos. Por otro lado, mientras que los pacientes del grupo CI iniciaban NP complementaria desde el primer día para alcanzar los objetivos, los pacientes del grupo estándar lo hicieron a partir del $7^{\circ}$ día si no se lograba el objetivo nutricional establecido. A pesar de que el grupo CI recibió más aporte calórico - proteico y tuvieron menos déficit calórico - proteico que el grupo estándar, no se encontró diferencia entre los dos grupos respecto a la calidad de vida física, la tasa de mortalidad, la estancia hospitalaria y las complicaciones infecciosas.

En la Tabla 5 se presenta un cuadro resumen comparativo de algunas características de los 3 estudios antes mencionados ${ }^{(19,21,22)}$ con la finalidad de ver lo heterogéneo de la población, de la metodología empleada y los resultados obtenidos. En ninguno de los tres estudios, los autores hacen referencia al estado o riesgo nutricional basal, e incluso en un estudio ${ }^{(22)}$ se excluyeron pacientes con malnutrición. Si bien la evidencia actual puede ser contradictoria por los estudios analizados ${ }^{(23-25)}$; la CI es el único método actualmente accesible y que puede ser usado con mayor confiabilidad en los pacientes en estado crítico $^{(1,25)}$. Esta herramienta clínica nos permite tener un parámetro objetivo de GE individualizado, monitorizar las variaciones que se pueden presentar en la evolución de la enfermedad crítica y nos ayudará al ajuste del aporte calórico de forma personalizada ${ }^{(1,25)}$. Su empleo en las unidades de cuidados críticos es necesario para la realización de ensayos randomizados controlados con la potencia estadística adecuada que nos proporcione nuevas luces de su utilidad ${ }^{(25)}$.

\section{PERSPECTIVAS}

Rattanachaiwong \& Singer ${ }^{(4)}$ realizaron una revisión narrativa sobre la utilidad que puede tener la CI con una Prueba en el Sitio de Atención (Point of Care Testing o POCT). Una POCT es una prueba de diagnóstico clínico aplicado en el lecho del paciente cuyos resultados afectan la toma de decisiones terapéuticas de manera rápida; por ejemplo, la pulsioximetría, la capnografía, y la glucometría, entre otras. Los autores concluyeron que la $\mathrm{CI}$, no solo es útil para medir el GE de forma puntual, sino que también sirve como guía del soporte metabólico en las diferentes etapas de la enfermedad - recuperación - agravamiento; por ejemplo, evaluando el grado de estrés generado por el daño tisular en pacientes postoperados, evitando la baja nutrición y la sobre nutrición en los pacientes en estado crítico. También puede ser útil, en pacientes no críticos con enfermedades crónicas, como herramienta de monitoreo que permita el diagnóstico precoz de intercurrencias, mala evolución y establecer el pronóstico; por ejemplo, la asociación de los resultados en la medición del GE con el pronóstico y sobrevida de pacientes con neoplasias malignas, cirrosis hepática, falla renal pacientes geriátricos.

Por otro lado, Gupta et al. ${ }^{(10)}$ describen el uso de la $\mathrm{CI}$ en una serie de estudios metabólicos donde se ha analizado la patogénesis de la obesidad, la influencia del polimorfismo genético en las enfermedades metabólicas y el papel de las intervenciones dietéticas con diferentes tipos de macronutrientes.

\section{RECOMENDACIONES}

Difundir los conocimientos básicos y aplicados de la CI, para así entender su relevancia clínica en el diagnóstico, manejo y monitoreo del estado metabólico del paciente en estado crítico.

\section{CONCLUSIÓN}

La calorimetría indirecta a pesar de los resultados contradictorios de algunos ensayos clínicos randomizados y controlados, continúa siendo una herramienta útil, 
Tabla 5. Comparación de las características clínicas de ensayos randomizados controlados donde la calorimetría indirecta guía los requerimientos energéticos en pacientes en estado crítico y se compara con las ecuaciones predictivas

\begin{tabular}{|c|c|c|c|c|c|c|}
\hline Estudio & \multicolumn{2}{|c|}{$\operatorname{TICACOS}^{(19)}$} & \multicolumn{2}{|c|}{ SPN ${ }^{(21)}$} & \multicolumn{2}{|c|}{ EAT-ICU(22) } \\
\hline Año de publicación & \multicolumn{2}{|l|}{2011} & \multicolumn{2}{|l|}{2013} & \multicolumn{2}{|l|}{2017} \\
\hline $\mathbf{N}^{\circ}$ de centros (país) & \multicolumn{2}{|l|}{1 (Israel) } & \multicolumn{2}{|l|}{2 (Suiza) } & \multicolumn{2}{|l|}{1 (Dinamarca) } \\
\hline Criterios de exclusión & \multicolumn{2}{|c|}{$\begin{array}{l}\text { Gestantes, trauma con coma, } \\
\text { cirrosis avanzada y cirugía } \\
\text { cardiaca abierta }\end{array}$} & \multicolumn{2}{|c|}{$\begin{array}{l}\text { Gestantes. } \\
\text { Readmitidos durante el estudio }\end{array}$} & \multicolumn{2}{|c|}{$\begin{array}{l}\text { Índice de masa corporal }<17 \\
\mathrm{~kg} / \mathrm{m}^{2} \\
\text { Aparentemente malnutridos }\end{array}$} \\
\hline Inicio de la intervención & \multicolumn{2}{|c|}{$\leq 48$ horas del ingreso a la $\mathrm{UCl}$} & \multicolumn{2}{|c|}{$4^{\circ}$ a $8^{\circ}$ día del ingreso a la UCI } & \multicolumn{2}{|c|}{$\leq 24$ horas del ingreso a la UCl } \\
\hline $\begin{array}{l}\text { Resultado primario de CI } \\
\text { versus EP }\end{array}$ & \multicolumn{2}{|c|}{$\begin{array}{l}\text { Tendencia a disminuir la } \\
\text { mortalidad por intención a tratar }\end{array}$} & \multicolumn{2}{|c|}{$\begin{array}{l}\text { Disminuyó las infecciones } \\
\text { nosocomiales y las infecciones } \\
\text { por paciente }\end{array}$} & \multicolumn{2}{|c|}{$\begin{array}{l}\text { No varió la calidad de vida física } \\
\text { a los } 6 \text { meses }\end{array}$} \\
\hline $\begin{array}{l}\text { Resultados secundarios } \\
\text { de } \mathrm{Cl} \text { versus EP }\end{array}$ & \multicolumn{2}{|c|}{$\begin{array}{l}\text { Aumentó los días en ventilación } \\
\text { y estancia en la UCI } \\
\text { Tendencia a aumentar las } \\
\text { neumonías asociadas al } \\
\text { ventilador }\end{array}$} & \multicolumn{2}{|c|}{$\begin{array}{l}\text { No afectó la estancia en la UCI ni } \\
\text { hospitalaria } \\
\text { No afectó la mortalidad }\end{array}$} & \multicolumn{2}{|c|}{$\begin{array}{l}\text { No afectó las infecciones } \\
\text { nosocomiales ni estancia } \\
\text { hospitalaria } \\
\text { No afectó la mortalidad a los } 28 \\
\text { días, } 90 \text { días y } 6 \text { meses }\end{array}$} \\
\hline $\begin{array}{l}\text { Método para calcular el } \\
\text { GE diario }\end{array}$ & $\mathrm{Cl}$ & EP & $\mathrm{Cl}$ & EP & $\mathrm{Cl}$ & EP \\
\hline $\begin{array}{l}\text { Número de pacientes } \\
\text { analizados }\end{array}$ & 65 & 65 & 153 & 152 & 100 & 99 \\
\hline Edad promedio en años & 59 & 62 & 61 & 60 & 63 & 68 \\
\hline $\begin{array}{l}\text { Índice de masa corporal } \\
\left(\mathrm{Kg} / \mathrm{m}^{2}\right)\end{array}$ & 27,8 & 27,4 & 25,4 & 26,4 & 22 & 22 \\
\hline $\begin{array}{l}\text { Puntaje promedio } \\
\text { APACHE II }\end{array}$ & 22,1 & 22,4 & 22 & 23 & - & - \\
\hline Puntaje promedio SOFA & 6,4 & 6,6 & - & - & 8 & 8 \\
\hline Puntaje promedio SAPS II & - & - & 49 & 47 & 47 & 48 \\
\hline $\begin{array}{l}\text { Inicio de NP } \\
\text { complementaria }\end{array}$ & \multicolumn{2}{|c|}{$\leq 48$ horas } & \multicolumn{2}{|c|}{$4^{\circ}$ a $8^{\circ}$ día } & $\leq 24$ horas & 70 día \\
\hline $\begin{array}{l}\text { Promedio del gasto } \\
\text { energético (Kcal) }\end{array}$ & 1976 & 1838 & 1892 & 1836 & 2069 & 1875 \\
\hline $\begin{array}{l}\text { Promedio de \% calórico } \\
\text { administrado }\end{array}$ & 105 & 80 & 103 & 77 & 97 & 64 \\
\hline $\begin{array}{l}\text { Promedio proteína } \\
\text { administrada (g / d) }\end{array}$ & $76 \mathrm{~g} / \mathrm{d}$ & $53 \mathrm{~g} / \mathrm{d}$ & $81 \mathrm{~g} / \mathrm{d}$ & $80 \mathrm{~g} / \mathrm{d}$ & 1,47 g / kg / día & 0,50 g / kg / día \\
\hline
\end{tabular}

UCI: Unidad de cuidados intensivos. GE: Gasto energético. Cl: Calorimetría indirecta. EP: Ecuación predictiva. NP: Nutrición parenteral.

confiable y accesible para el manejo nutricional de los pacientes en estado crítico. Esta herramienta ayuda a realizar un manejo dirigido a objetivos, reajuste nutricional de acuerdo con la evolución clínica de forma individualizada y de esta manera evitaría la sub o sobre nutrición con los resultados adversos relacionados con ellas.

\section{Financiamiento}

El presente estudio no tuvo financiación.

\section{Conflicto de intereses}

El autor declara no tener conflicto de intereses. 


\section{Referencias bibliográficas}

1. De Waele E, Honoré PM, Malbrain MLNG. Does the use of indirect calorimetry change outcome in the ICU? Yes it does. Curr Opin Clin Nutr Metab Care. 2018;21(2):126-29. doi: 10.1097/MCO.0000000000000452.

2. McClave SA, Taylor BE, Martindale RG, Warren MM, Johnson DR, Braunschweig C, et al. Guidelines for the Provision and Assessment of Nutrition Support Therapy in the Adult Critically Ill Patient: Society of Critical Care Medicine (SCCM) and American Society for Parenteral and Enteral Nutrition (A.S.P.E.N.). JPEN J Parenter Enteral Nutr. 2016;40(2):159-211. doi: 10.1177/0148607115621863.

3. Singer P, Blaser AR, Berger MM, Alhazzani W, Calder PC, Casaer MP, et al. ESPEN guideline on clinical nutrition in the intensive care unit. Clin Nutr. 2019;38(1):48-79. doi: 10.1016/j.clnu.2018.08.037.

4. Rattanachaiwong S, Singer P. Indirect calorimetry as point of care testing. Clin Nutr. 2019;38(6):2531-44. doi: 10.1016/j. clnu.2018.12.035.

5. Zusman O, Theilla M, Cohen J, Kagan I, Bendavid I, Singer P. Resting energy expenditure, calorie and protein consumption in critically ill patients: a retrospective cohort study. Crit Care. 2016;20(1):367. doi: 10.1186/s13054-016-1538-4.

6. Yatabe T. Strategies for optimal calorie administration in critically ill patients. J Intensive Care. 2019;7:15. doi: 10.1186/ s40560-019-0371-7.

7. Heymsfield SB, Bourgeois B, Thomas DM. Assessment of human energy exchange: historical overview. Eur J Clin Nutr. 2017; 71(3):294-300. doi: 10.1038/ejcn.2016.221.

8. Oshima T, Berger MM, De Waele E, Guttormsen AB, Heidegger CP, Hiesmayr M, et al. Indirect calorimetry in nutritional therapy. A position paper by the ICALIC study group. Clin Nutr. 2017;36(3):651-662. doi: 10.1016/j. clnu.2016.06.010.

9. Marino PL, Sutin KM. Necesidades de sustratos metabólicos. En: Marino PL, Sutin KM. El libro de la UCI. $3^{\text {a }}$ ed. Barcelona: Lippincott Williams \& Wilkins; 2008. p. 775-89.

10. Gupta RD, Ramachandran R, Venkatesan P, Anoop S, Joseph M, Thomas N. Indirect Calorimetry: From Bench to Bedside. Indian J Endocrinol Metab. 2017; 21(4):594-9. doi: 10.4103/ ijem.IJEM_484_16.

11. Mtaweh H, Tuira L, Floh AA, Parshuram CS. Indirect Calorimetry: History, Technology, and Application. Front Pediatr. 2018;6:257. doi: 10.3389/fped.2018.00257.

12. Ahmad D, Joseph K, Halpin C. Nutrition and Indirect Calorimetry [internet]. En: Hoag J. Oncology Critical Care. Londres: IntechOpen; 2016 [citado el 15 de Julio del 2019]. Disponible en: https://www.intechopen.com/books/oncology-critical-care/nutrition-and-indirect-calorimetry.

13. Marsé Milla P, Diez Poch M, Raurich Puigdevall JM. Calorimetría: aplicaciones y manejo. Nutr Clin Med [inter- net]. 2008 [Consultado 05 feb 2020];Vol.II(3):155-166. Disponible en: http://www.nutricionclinicaenmedicina. com/images/flash/nutr080303/files/assets/basic-html/ page1.html.

14. American Association for Respiratory Care. Metabolic Measurement Using Indirect Calorimetry During Mechanical Ventilation-2004 Revision \& Update. Respiratory Care. 2004;49(9):1073-1079 [Internet]. [Fecha de consulta: 02 de junio del 2019] Disponible en: https://www.aarc.org/wpcontent/uploads/2014/08/09.04.1073.pdf.

15. Delsoglio M, Achamrah N, Berger MM, Pichard C. Indirect Calorimetry in Clinical Practice. J Clin Med. 2019; 8(9): 1387. doi: $10.3390 /$ jcm8091387.

16. Zusman O, Kagan I, Bendavid I, Theilla M, Cohen J, Singer P. Predictive Equations versus Measured Energy Expenditure by Indirect Calorimetry: A Retrospective Validation. Clin Nutr. 2019;38(3):1206-10. doi: 10.1016/j.clnu.2018.04.020.

17. Critical Care Nutrition: Sistemic Reviews. Nutritional Prescription: Use of Indirect Calorimetry vs. Predictive Equations [internet]. Ontario: Critical Care Nutrition; 2018. [Fecha de consulta: 15 de Julio del 2019] Disponible en: https://www.criticalcarenutrition.com/docs/systematic_ reviews_2018/3.1\%20Indirect\%20Calorimetry_2018.pdf.

18. Saffle JR, Larson CM, Sullivan J. A randomized trial of indirect calorimetry-based feedings in thermal injury. J Trauma. 1990;30(7):776-82. doi: 10.1097/00005373-19900700000003.

19. Singer P, Anbar R, Cohen J, Shapiro H, Shalita-Chesner M, Lev $S$, et al. The tight calorie control study (TICACOS): a prospective, randomized, controlled pilot study of nutritional support in critically ill patients. Intensive Care Med. 2011;37(4):601-9. doi: 10.1007/s00134-011-2146-z.

20. Oshima T, Graf S, Heiddeger CP, Genton L, Pugin J, Pichard C. Can calculation of energy expenditure based on $\mathrm{CO}_{2}$ measurements replace indirect calorimetry? Crit Care. 2017;21:13. doi: 10.1186/s13054-016-1595-8.

21. Heidegger CP, Berger MM, Graf S, Zingg W, Darmon P, Costanza MC, et al. Optimisation of energy provision with supplemental parenteral nutrition in critically ill patients: a randomised controlled clinical trial. Lancet. 2013; 381(9864): 385-93. doi: 10.1016/S0140-6736(12)61351-8.

22. Allingstrup MJ, Kondrup J, Wiis J, Claudius C, Pedersen UG, Hein-Rasmussen R, et al. Early goal-directed nutrition versus standard of care in adult intensive care patients: the singlecentre, randomised, outcome assessor-blinded EAT-ICU trial. Intensive Care Med. 2017; 43(11):1637-47. doi: 10.1007/ s00134-017-4880-3.

23. Casaer MP, Reignier J, Doig G. Optimal guidance for early nutrition therapy in critical illness? Intensive Care Med. 2017; 43(11):1720-22. doi: 10.1007/s00134-017-4881-2.

24. Singer P, Berger MM, Weijs PJM. The lessons learned from the EAT ICU study. Intensive Care Med. 2018; 44(1):133-34. doi: 10.1007/s00134-017-4977-8. 
25. Wernerman J, Christopher KB, Annane D, Casaer MP, Coopersmith CM, Deane AM, et al. Metabolic support in the critically ill: a consensus of 19. Crit Care. 2019; 23(1):318. doi: 10.1186/s13054-019-2597-0.
26. Pereira Cunill JL, Garrido Vázquez M. Interpretación de la Calorimetría Indirecta. [Blog en Internet] [Fecha de consulta: 01 de junio del 2019] Disponible en: https://es.slideshare. net/jlpc1962/interpretacion-de-la-calorimetra-indirecta. 